\title{
OFF-LINE VS. ON-LINE CALIBRATION OF A PANORAMIC-BASED MOBILE MAPPING SYSTEM
}

\author{
Bertrand Cannelle, Nicolas Paparoditis, Marc Pierrot-Deseilligny, Jean-Pierre Papelard \\ IGN, MATIS \\ 73 Avenue de Paris, 94160 Saint-Mandé, France; \\ Université Paris-Est \\ firstname.lastname@ign.fr
}

KEY WORDS: mobile mapping system, photogrammetry, multi-camera panoramic head, calibration, off-line calibration, on-line calibration, panoramic head calibration with very low image overlap, bundle adjustment.

\begin{abstract}
:
This paper presents two methods to calibrate a photogrammetric-based mobile mapping system.

The paper is organised as follow. The first part presents the problem we are trying to solve, i.e. the calibration of multi-camera head on a mobile mapping system equipped with an Inertial Navigation System (INS). We want to estimate on the one hand the relative pose of images within the head and on the other hand the relative pose between the head and the INS The second part presents the first calibration method which is based on observations of a sub-millimeter accuracy topometric target network. The third part explores a method to determine the calibration on-line, i.e. using the images acquired during the surveys. The final section compare the two methods on an neutral data set and resumes the pros and cons of each method.
\end{abstract}

\section{INTRODUCTION}

Mobile mapping systems have encoutered a large success in the recent years with wide applications ranging from 3D metrology to multimedia as evidenced by the work of (Ellum and El-Sheimy, 2002) or even more recently in (Petrie, 2010). Most of these systems are LiDAR-based for metrology and inventory for roadbased applications. Nevertheless some other systems uses optical devices for surveying. Some use stereo-rigs in order to provide $3 \mathrm{D}$ photogrammetric plotting services or to ease automatic $2 \mathrm{D}$ or 3D object extraction from images. Some other systems such as the Google and the EarthMine cars are panoramic-based in order to propose immersive visualisation possibilities often on the web. Nevertheless, images are not used so much yet for 3D metrology. For the applications, where the accuracy of the 3D maps or 3D object-based databases generated from the optical imagery are crucial, e.g. 3D surveying for civil engineering and 3D maps or 3D visual landmarks databases for autonomous navigation, an accurate geometric calibration of the system is mandatory. In order to achieve such a calibration, one needs to master on the one hand internal geometric calibration of all sensors, and on the other hand the relative pose estimation of all sensors with one the other and the relative pose of the set of sensors with respect to the reference frame of the vehicle they are mounted on.

In this paper we aim at calibrating a multi-camera panoramic head on a vehicle, i.e. to determine the relative orientation of all cameras composing the panoramic head with respect to the vehicle. Our panoramic head can not be calibrated as a whole by itself due to the fact that the the overlapp between the images is very small (a few pixels). We will thus aim at estimating independtly the extrinsic parameters of each camera composing the camera. We will describe and compare two complete calibration processes to estimate the pose of cameras (composing a panoramic head) relatively to the vehicle they are mounted on. The first, called the off-line calibration, is carried out independtly from the surveys and needs a topometric target network and a manual and/or automatic plotting of target observations in the images. The second, called the on-line calibration, is carried out directly on the survey data. Both methods will be compared.

\section{DESCRIPTION OF OUR MOBILE-MAPPING VEHICLE}

In this section and in Figure 1, we present our photogrammetricbased Mobile Mapping Sytem Stéréopolis V2.

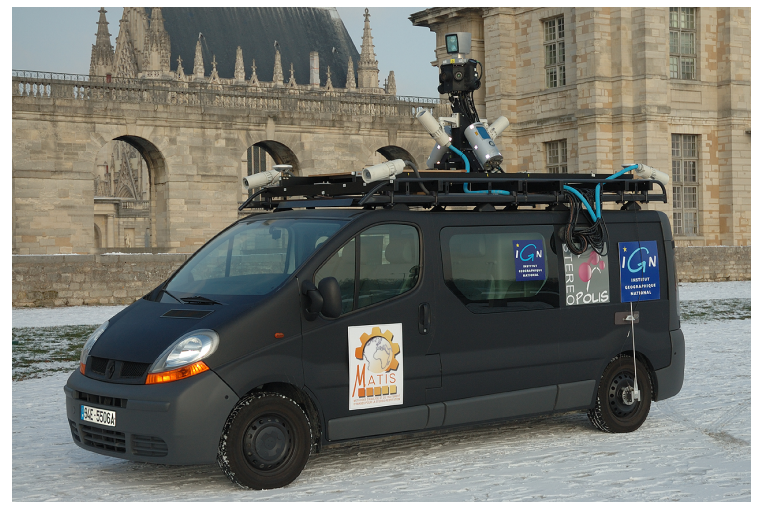

Figure 1: Stéréopolis V2

\subsection{The panoramic head}

Our panoramic head is based on a set of 10 full HD PIKE cameras with high quality lenses (no chromatic aberrations on the image field). These scientific cameras are higly synchronisable, i.e. all images captured within a panoramic are acquired at the same time thus avoiding parallax problems. These cameras have also with very high dynamics (close to 12 bits) to capture imagery with all lighting conditions (from hard shadows to bright sun exposures). All the cameras of the head acquire images at the same aperture for each pose and all along the survey. Moreover, the exposure time is small to avoid blur due to the vehicle displacement. The images which are captured have a very high angular resolution, i.e. 1 pixel correesponds to 0.04 degrees.

Our panoramic head has been designed to limit the parallax between the different perspective centers. Nevertheless, the parallax can not be considered negligible thus we use the position 
of the perspective centers corresponding the CAD design plans. The perspective center is the position where all perspective beams converge and is very difficult to estimate in theory as it does not correspond to one unique point in reality in a complex optics (McGlone et al., 2004). We thus measure the position of the entry nodal point assumed to be equal to the perspective center with respect to object space with a well-known ad-hoc optical alignment technique. The idea is to put the camera on a pan-tilt system and to capture series of photos with near and far objects by pan-tilting the camera. If the relative order between the objects changes between the images, the camera is not well centered and the camera body is shifted manually until convergence.

\subsection{The positioning system}

An inertial Navigation System hybridating, in a tightly-coupled way, the data of two GPS, an Inertial Measurement Unit (IMU), and an odometer provides the position and an accurate orientation of the vehicle in a global reference system even when GPS is masked or corrupted by multiple paths. During GPS gaps, the absolute position reconstructed from the IMU only is biased due to its drifts. The trajectography data acquired on-line by the INS is processed a posteriori in both forward and reverse directions to smooth trajectory and reduces the errors due to lacks of GPS signal. The IMU is mounted rigidely at the foot of the panoramic multi-head camera in order to avoid level arm errors (the wider the level arm, the higher the impact on position and rotation errors).

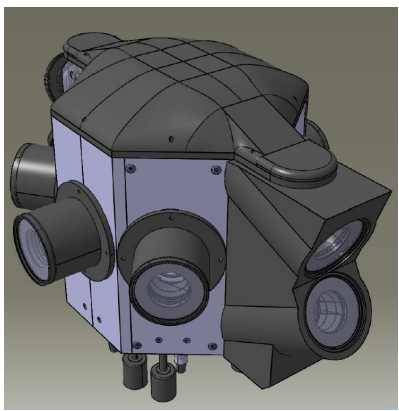

a

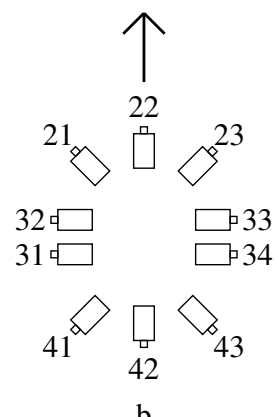

b
Figure 2: (a) Panoramic head mounted on mobile mapping system. (b) Id of cameras used

For orientations, we can not use the CAD design plan as a reference. Indeed on the one hand the optical axis of each camera is different and on the other the impact of a mechanical errors is non negligible. For example, for a $4 \mathrm{~cm}$ camera body size, a 0.1 $\mathrm{mm}$ drilling error on the camera fixation holes induce an error of 2.5 pixels on the center of the images and 8 on the border of the images. Nevertheless, these CAD orientions can be used as initial solutions for the linearisation of the bundle equations.

\section{CALIBRATION ON A TOPOMETRIC TARGET NETWORK}

This section first introduces the conventions and systems of coordinates used throughout the paper. Then, the calibration process of the mobile mapping system is detailed. Finally experimentations are presented.

In this part, almost figures contains 3D model. They serve only for illustrations but are not used as input to process.

\subsection{Notations and Conventions}

Integrating sensors to a mobile platform requires to define several coordinate systems:

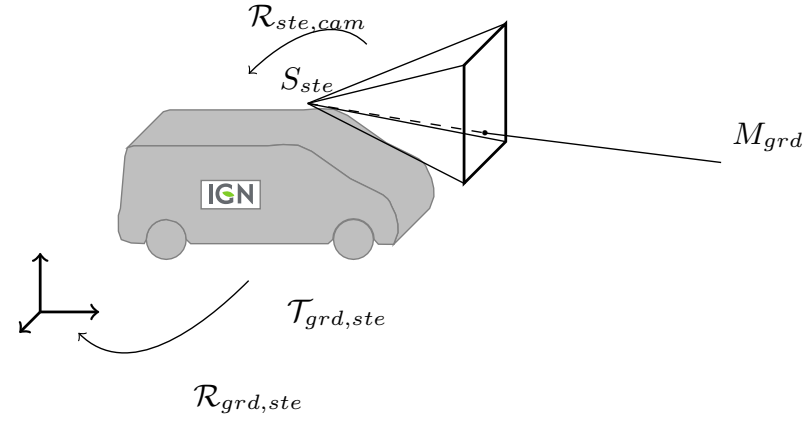

Figure 3: Systems of coordinates used

- Ground reference system ( $g r d$ )

- Reference system of the mobile mapping system (ste) defined by a rotation $\mathcal{R}_{\text {grd,ste }}$ and a translation $\mathcal{T}_{\text {grd,ste }}$ in ground coordinates

- Reference system of a camera ( $\mathrm{cam}$ ) defined by a rotation $\mathcal{R}_{\text {ste,cam }}$ and a translation $\mathcal{T}_{\text {ste,cam }}$.

Figure 3 represents theses different systems. If we call $M_{x x x}$ the coordinates of a point $M$ in the $x x x$ coordinate system, the change of coordinates are expressed as:

$$
\begin{aligned}
M_{\text {ste }} & =\mathcal{R}_{\text {grd }, \text { ste }}\left(M_{\text {grd }}-\mathcal{T}_{\text {grd }, \text { ste }}\right) \\
M_{\text {sen }} & =\mathcal{R}_{\text {ste }, \text { cam }}\left(M_{\text {ste }}-\mathcal{T}_{\text {ste }, \text { cam }}\right)
\end{aligned}
$$

In "classical" photogrammetry (McGlone et al., 2004), a 3D point $M_{g r d}$ in object space projects itself in the image space of a camera with coordinates $(c, l)$ by:

$$
\left(\begin{array}{c}
c \\
l
\end{array}\right)=\left(\begin{array}{c}
c_{P P A} \\
l_{P P A}
\end{array}\right)-p \frac{\mathcal{R}_{g r d, c a m}\left(M_{g r d}-S_{g r d}\right)}{{ }^{t} \mathbf{k} \mathcal{R}_{\text {grd,cam }}\left(M_{\text {grd }}-S_{\text {grd }}\right)}
$$

where:

- $p$ : the focal lenght of the camera

- $\left(c_{P P A}, l_{P P A}\right):$ the principal point of auto collimation in image space

- $\mathbf{k}$ the third unitary vector of the ground system coordinate

- $S_{g r d}$ the position of the center of the camera in the object space and $\mathcal{R}_{\text {grd,cam }}$ the rotation of the camera with the object space.

Equations (1), (2) and (3) can finally be combined to build (4), which is formula to obtain the image measurement in camera $i$ of a point $M$ in the ground reference frame.

Unlike the approch of (Scheller et al., 2007) who calibrates at the same time the intrinsic and extrinsic parameters, we choose to calibrate each camera separately following a process similar to (Tsai, 1987) and (Hartley and Zisserman, 2000). We consider a radial polynomial distortion model of the form:

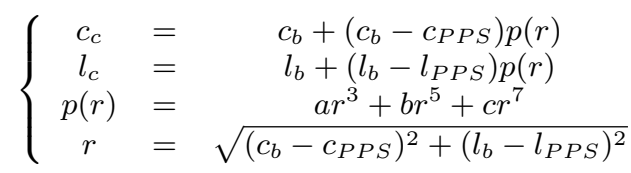

where $(c b, l b)$ is the measure taken directly in image space and $(c c, l c)$ is the corrected measurement. Note that the Principal Point of Symmetry $\left(c_{P P S}, l_{P P S}\right)$ which is the intersection of the optical axis with the image focal plane may differ from the Principal Point of Autocollimation $\left(c_{P P A}, l_{P P A}\right)$. 


$$
\left(\begin{array}{c}
c \\
l
\end{array}\right)_{\mathbf{i}, \mathbf{j}}=\left(\begin{array}{c}
c_{P P A} \\
l_{P P A}
\end{array}\right)_{\mathbf{i}}-p_{\mathbf{i}} \frac{\mathcal{R}_{s t e_{\mathbf{j}}, c a m_{\mathbf{i}}} \mathcal{R}_{g r d, s t e_{\mathbf{j}}}\left(M_{g r d}-\left(\mathcal{R}_{\text {cam }_{\mathbf{i}}, s t e_{\mathbf{j}}} S_{\text {cam }_{\mathbf{i}}}+\mathcal{T}_{s t e_{\mathbf{j}}, g r d}\right)\right)}{{ }_{\mathbf{k} \mathcal{R}_{s t e_{\mathbf{j}}}, c_{m_{\mathbf{i}}}} \mathcal{R}_{g r d, s t e_{\mathbf{j}}}\left(M_{g r d}-\left(\mathcal{R}_{c a m_{\mathbf{i}}, s t e_{\mathbf{j}}} S_{c a m_{\mathbf{i}}}+\mathcal{T}_{s t e_{\mathbf{j}}, g r d}\right)\right)}=f_{\mathbf{i}, \mathbf{j}}\left(M_{g r d}\right)
$$

\subsection{Calibration process}

One can see in formula 4 that both positions and orientations intervene. To initialize our photogrammetric bundle we use as initial solutions the orientations and positions of cameras corresponding to the CAD design of the vehicle. In the compensation we only allow positions of the perspective centers to be very close to the ones of the design (one to a few centimeters). Using these strong observations are important as the positions can not be determined as precisely by the bundle adjustment itself. The orientation interval is set much looser as the orientation of the optical axis of each camera depends on the quality of the alignment of the lenses and on the mechanical alignment of the optics on the camera body.

\subsection{Calibration on a surveyed 3D target network}

We have show a topometry method to determine with precision the position of the camera but we have demonstrated it's difficult to determine the rotation. Now a method will be presented to find the best positions and rotations of the camera. This is a method on a topometric target network which needs an adapted place.

Our target network is a facility where the mobile mapping system can move and capture images of 3D surveyed targets. These targets are measured with a sub-millimeter accuracy with precise surveying techniques. The observation of the $3 \mathrm{D}$ targets in the images is carried out manually by an operator (but this could also be made automatically with specific coded targets).

Figure 4 presents four positions of Stéréopolis V2 with the multicamera images which have been acquired and the 3D rays corresponding to the image observations of targets.

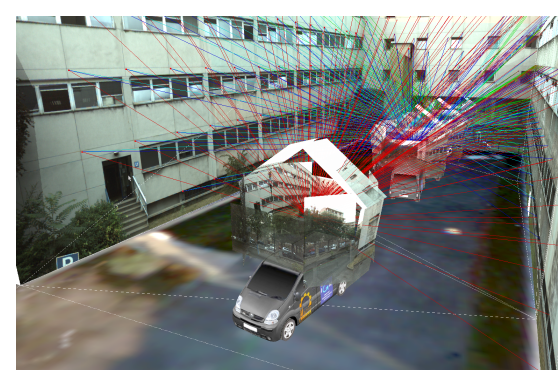

a

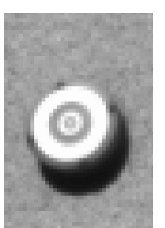

b
Figure 4: (a) Representation of Stéréopolis V2 during calibration phase. Image plans (in transparent) and measurements(lines) are also visible. (b) Example of target

\subsection{Mathematical formulation of the problem}

In formula 4 , the equation explains the link between points in the ground reference system and corresponding measurements in image space. So with a set of Ground Control Points (GCP) (noted $M_{\text {grd }}$ in equation), and corresponding image measurements, we propose to calibrate our system using a method similar to the bundle adjustment presented in (Schmid, 1959) and (Triggs et al., 2000) and used by (Scheller et al., 2007) in the mobile mapping calibration context. Formula 5 presents the system to minimize which is solved by least squares and bundle adjustment. To initialize the system, we use $\mathcal{T}_{\text {grd,ste }}, \mathcal{R}_{\text {grd,ste }}$ with information given by positionning system and $\mathcal{S}_{\text {cam }}, \mathcal{R}_{\text {ste }, \text { cam }}$ with CAD

$$
\begin{aligned}
& \underset{\left[\mathcal{S}_{\text {cam }}, \mathcal{R}_{\text {ste }, \text { cam }}\right]_{i=1}^{\# N c}}{\arg \min } \sum_{k}^{N m} \sum_{i}^{N c} \sum_{j}^{N p}\left(f_{i, j}-\left(\begin{array}{c}
c_{k} \\
l_{k}
\end{array}\right)_{i, j}\right)^{2} \\
& {\left[\mathcal{T}_{\text {grd }, \text { ste }}, \mathcal{R}_{\text {grd }, \text { ste }}\right]_{j=1}^{\# N p}}
\end{aligned}
$$

with :

- $N m$ is the number of image measurments.

- $N c$ is the number of camera on the mobile mapping vehicle.

- $N p$ is the number of position of the mobile mapping vehicle.

- ${ }^{T}(c, l)_{i, j}$ is the measure $k$ in image taken by camera $i$ in the pose $j$ of the MMS.

\subsection{Experimentations}

We have choose 4 positions of the vehicle to calibrate the system. We have manually measured 534 target projections in the different images. Figure 5 presents a bird's view of the geometric configuration of our experimentation.

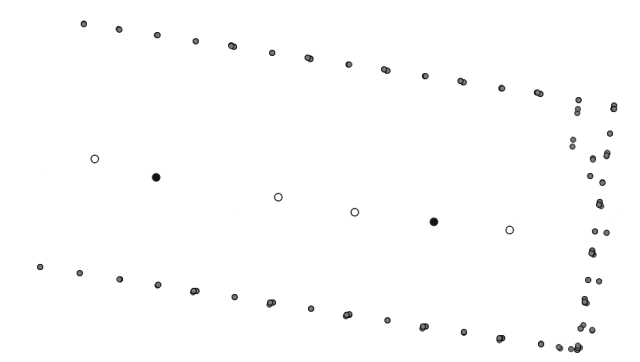

Figure 5: Configuration and distribution of image poses and targets. In gray: point known in $3 \mathrm{D}$, in white: position of the vehicle used for calibration, in black: position of the vehicle used for control.

3.5.1 Quantitative results On table 1, we can see the difference between with no calibration and with calibration. Histogram on figure 6 describes the repartition of norm of measurements as a function of residuals. Most of image residues smaller than 1 pixel. Mean is 0.47 pixels and standard deviation is 0.37 pixels. By contruction,the sum of the residues of images measurments is null. We all observe that the sum of residues per camera is also null whichs proves there is no biais in the estomation.

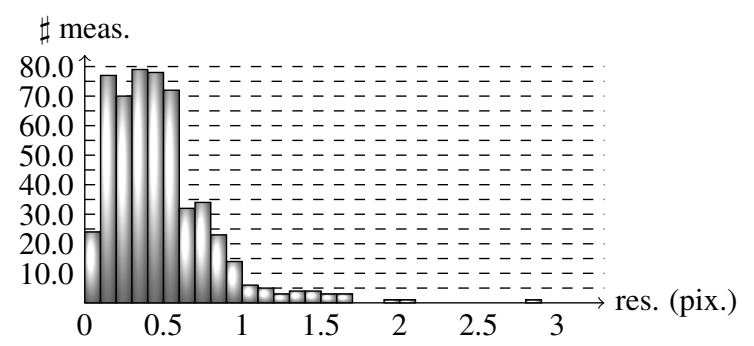

Figure 6: Repartition of residues on image measurements after calibration 


\begin{tabular}{|c||c|c|c|c|}
\hline \multicolumn{1}{|c||}{ Cameras } & \multicolumn{2}{c|}{ Before Calib. (pix.) } & \multicolumn{2}{c|}{ After Calib. (pix.) } \\
& mean & std. dev. & mean & std. dev. \\
\hline \hline 21 & 6.10 & 1.31 & 0.41 & 0.25 \\
22 & 3.47 & 1.99 & 0.76 & 0.41 \\
23 & 7.52 & 3.63 & 0.43 & 0.25 \\
31 & 18.67 & 2.50 & 0.25 & 0.14 \\
32 & 15.27 & 1.79 & 0.57 & 0.39 \\
33 & 8.16 & 2.02 & 0.35 & 0.22 \\
34 & 22.12 & 1.91 & 0.44 & 0.25 \\
41 & 6.00 & 2.24 & 0.49 & 0.31 \\
42 & 2.21 & 1.09 & 0.35 & 0.22 \\
43 & 5.00 & 0.89 & 0.41 & 0.19 \\
\hline
\end{tabular}

Table 1: Norm of residues on images measurements per cameras before and after calibration. std. dev. (standart deviation).

3.5.2 Qualitative results On figure 7 one can see one of the (surveyed) facades of the facility textured with the images acquired by our MMS. The images are projected on the 3D plane of the facade which is in the same reference system as the targets. Before calibration, windows are not aligned, and windows edges are discontinuous on each side of the mosaicquing. After calibration windows are aligned and egdes are perfectly continuous. This means that the relative pose of the cameras are correctly estimated.

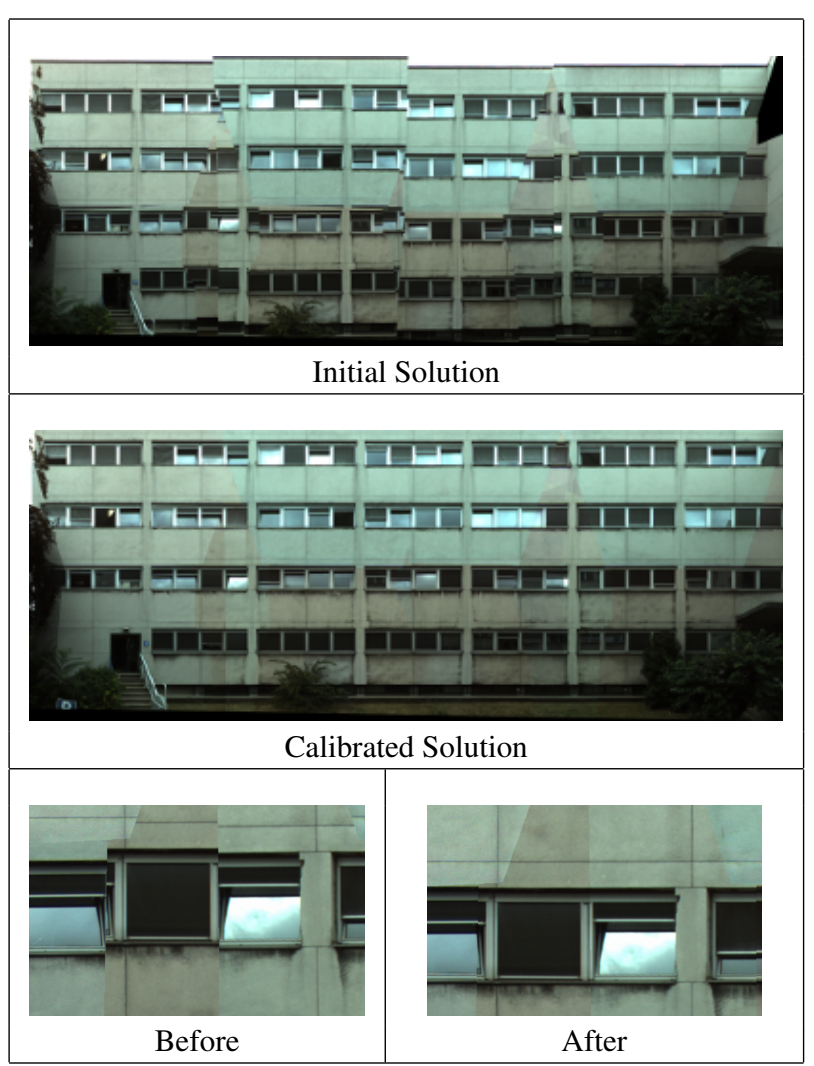

Figure 7: Comparison of reprojection of images on plan before and after calibration

3.5.3 Control To control the result of the calibration, we used on the same facility two extra poses of the MMS with 188 measurements on the images. We only estimate the pose of the vehicle by bundle adjustment and we use as position and rotation of cameras the results of process presented in 3.2. Only 3 targets are used as GCPs and the others are used as check point

On figure 8 and $n$ figure 9 , one can see respectively the repartition of residues on this check dataset without taking into account the calibration results and the repartition of residual on check dataset with positions and rotations of cameras after calibration.

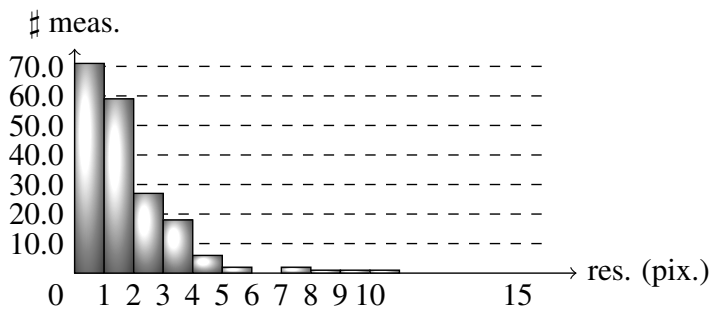

Figure 8: Repartition of residuals on measurements without taking into account the calibration on control dataset

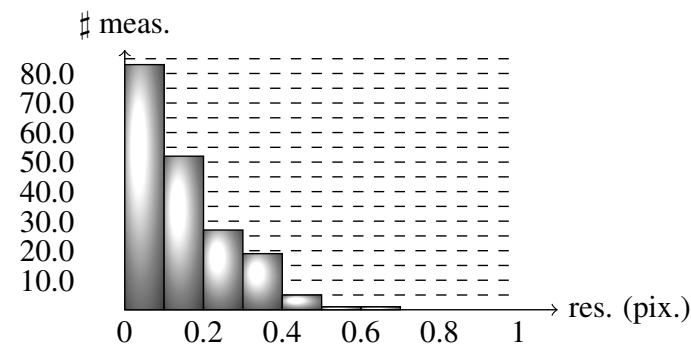

Figure 9: Distribution of measurement residues when taking into account the calibration on the control dataset

The residues on image measurements after calibration have a mean of 0.15 pixel and a standard deviation of 0.12 pixel. The error on the CPs has a mean of $0.016 \mathrm{~m}$ and a standard deviation of $0.012 \mathrm{~m}$. This very good result allows us to conclude that our calibration process on a topometric target network is a good solution to calibrate our mobile mapping system with a good precision.

\section{ON-LINE CALIBRATION}

The main drawback of the first method is that one must first have a facility to perform the calibration and an operator to measure the position of targets on images if the target detection and localisation is not automatic. That is why we have developped a second method to compute the calibration without GCPs and in a fully automatic way.

\subsection{Automatic measures used for the on-line calibration}

To compute an on-line calibration, we need a set of consecutive multi-camera panoramas acquired by our MMS. Then, interest points are extracted and matched between images (in this paper, we use SIFT (Lowe, 2004)). Outliers are automatically eliminated by classical photogrammetric filtering (elimination of measurements with a hight residual before compensation). Tie points are matched across differetns cameras for different vehicle position's.

\subsection{Mathematical formulation of the problem}

In formula 4 , the equation describes the link between points in the ground reference system and measurements in image space. In the case of an on-line calibration, the position of ground points are not known. That is why in Formula 6, we can see three groups of unknowns. First, the position and rotation of camera relatively to the vehicle/INS. Second the position and rotation of vehicle relatively to the ground. Third the position of tie points relatively to the ground. That is why we have one more term in the minimization relative to Equation 3. This term corresponds to 
the minimisation of the position of the $3 \mathrm{D}$ points which are not known.

$$
\begin{gathered}
\underset{\left[\mathcal{S}_{\text {cam }}, \mathcal{R}_{\text {ste }, \text { cam }}\right]_{i=1}^{\# N c}}{\arg \min } \sum_{i}^{N c} \sum_{j}^{N p} \sum_{k}^{N m} \sum_{n}^{N i}\left(f_{i, j}\left(M_{k}\right)-\left(\begin{array}{c}
c_{n} \\
l_{n}
\end{array}\right)\right)^{2} \\
{\left[\mathcal{T}_{\text {grd }, \text { ste }}, \mathcal{R}_{\text {grd }, \text { ste }}\right]_{j=1}^{\# N p}} \\
{\left[\mathcal{M}_{k}\right]_{k=1}^{\# N m}}
\end{gathered}
$$

with : $N i$ is the number of image measurments, $N c$ is the number of camera on the mobile mapping vehicle, $N p$ ) is the number of position of the mobile mapping vehicle, $N m$ is the number of tie point. The minimisation is made by least square and bundle adjustment. To initialize the system we use $\mathcal{T}_{\text {grd,ste }}, \mathcal{R}_{\text {grd,ste }}$ with information given by positionning system and $\mathcal{S}_{\text {cam }}, \mathcal{R}_{\text {ste,cam }}$ with CAD. The scale of the bundle adjustment has been fixed by using initial values given by GPS/INS system. Then for each iteration $i$, we use a mathematical constraint on position of vhicle $\left(P_{v, i}\right)$ such as $P_{v, i}=\lambda P_{v, i+1}$ with $\lambda$ small. This constraint is necessary to avoid a divergence of the calculation

\subsection{Experimentation}

We have chosen 30 poses of the vehicle to calibrate our system. We have automatically generated 10756 tie points with the SIFT detector in all images. Figure 10 presents the geometric configuration of our experimentation. After minimisation we obtain a mean residue of 0.29 pixels on all image measurements with a standard deviation of 0.40 pixels.

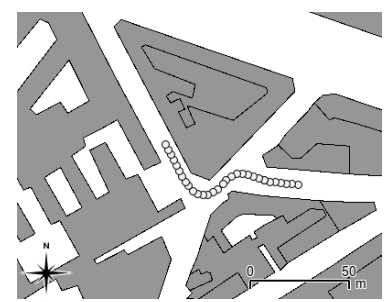

a

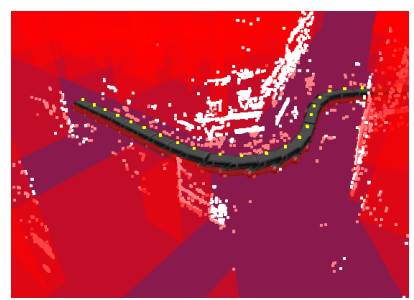

b
Figure 10: Geometric configuration used for the on-line calibration. (a) the vehicle trajectory and poses on a 2D map. (b) Visualisation in 3D. In white : the 3D tie points, in black: the vehicle poses, in red: a 3D model of the scene which is just shown to demonstrate that the 3D tie points are reliable and correspond to structures of the scene

\subsection{Control}

To control the result of the calibration, we use the same data set has the one used in 3.4 .3 for the off-line method (i.e. 2 positions of the vehicle and with 188 measurements on images) but with the on-line method. On figure 11, one can see a distribution of residual on check dataset with taking into account the on line calibration results. If we compare this histogram with histogram of figure 8 , we can conclude that on-line calibration method improves our model by reducing image residues.

\section{COMPARISON OF THE TWO CALIBRATION METHODS}

In this section we present a comparison between the results of offline and on-line calibration. First, we will focus on the residues in image space and then we will focus on the residues in 3D.

To compare the two methods, we consider another data set with : 4 consecutive panoramas acquired the mobile mapping system,

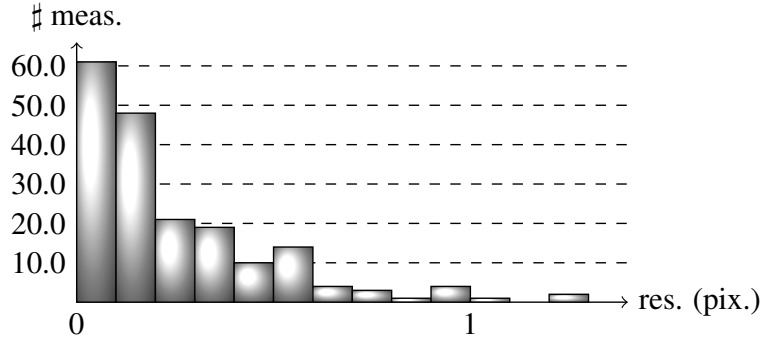

Figure 11: Distribution of image measurement residuals with the on-line calibration results

14 Ground Control Points (GCP) and 8 Check Points (CP). The GCPs and CPs are natural urban details (corner of windows, center of a road signs, etc.) have been measured by topometric techniques. The absolute accuracy of these surveyed $3 \mathrm{D}$ points is better than $1 \mathrm{~cm}$.

We estimate through a bundle adjustment the absolute and relative poses (position and orientation) of the panoramas with ground control points and with respectively the calibration computed with the off-line and the on-line method.

\subsection{Residuals in image space an in object space}

Table 2 presents a comparison between the results obtained with no calibration (CAD design) and each of the two methods. First it is obvious that a photogrammetric-based calibration is necessary to obtain a very good precision on the image models and low image measurement residues. The two photogrammetric methods provide pretty similar results. The residues on check points $(0.2)$ are obviously higher than GCPs because GCPs are compensated whereas check points are not.

\begin{tabular}{|c|cccc|}
\hline & \multicolumn{4}{|c|}{ Ground Control Point } \\
& Max & Med & Mea & Std.Dev. \\
\hline without & 11.75 & 2.13 & 3.96 & 3.88 \\
\hline off line & 1.43 & 0.52 & 0.54 & 0.27 \\
\hline on line & 1.49 & 0.50 & 0.55 & 0.30 \\
\hline \hline & \multicolumn{4}{|c|}{ Check point } \\
& Max & Med & Mea & Std.Dev. \\
\hline without & 6.73 & 1.45 & 2.11 & 1.83 \\
\hline off line & 0.95 & 0.26 & 0.30 & 0.19 \\
\hline on line & 1.04 & 0.28 & 0.32 & 0.21 \\
\hline
\end{tabular}

Table 2: Residual on images measurements on ground control points and on check point (in pix.). Max (maximum), Med (Median), Mea (mean), Std.Dev(Standart Deviation)

\begin{tabular}{|c|cccc|}
\hline & \multicolumn{4}{|c|}{ Ground Control Point } \\
& Max & Med & Mea & Std.Dev. \\
\hline without & 0.125 & 0.020 & 0.030 & 0.029 \\
\hline off line & 0.012 & 0.004 & 0.005 & 0.003 \\
\hline on line & 0.013 & 0.004 & 0.005 & 0.004 \\
\hline \hline & \multicolumn{4}{|c}{ Check point } \\
& Max & Med & Mea & Std.Dev. \\
\hline without & 1.034 & 0.269 & 0.396 & 0.350 \\
\hline off line & 0.092 & 0.040 & 0.044 & 0.023 \\
\hline on line & 0.032 & 0.025 & 0.023 & 0.009 \\
\hline
\end{tabular}

Table 3: Residual on ground control points and on check point (in meters). Max (maximum), Med (Median), Mea (mean), Std.Dev(Standart Deviation)

Table 3 presents the results between the methods in object space. One can see that the results which are achieved are very good On GCPs one can see that there is no significant difference between 


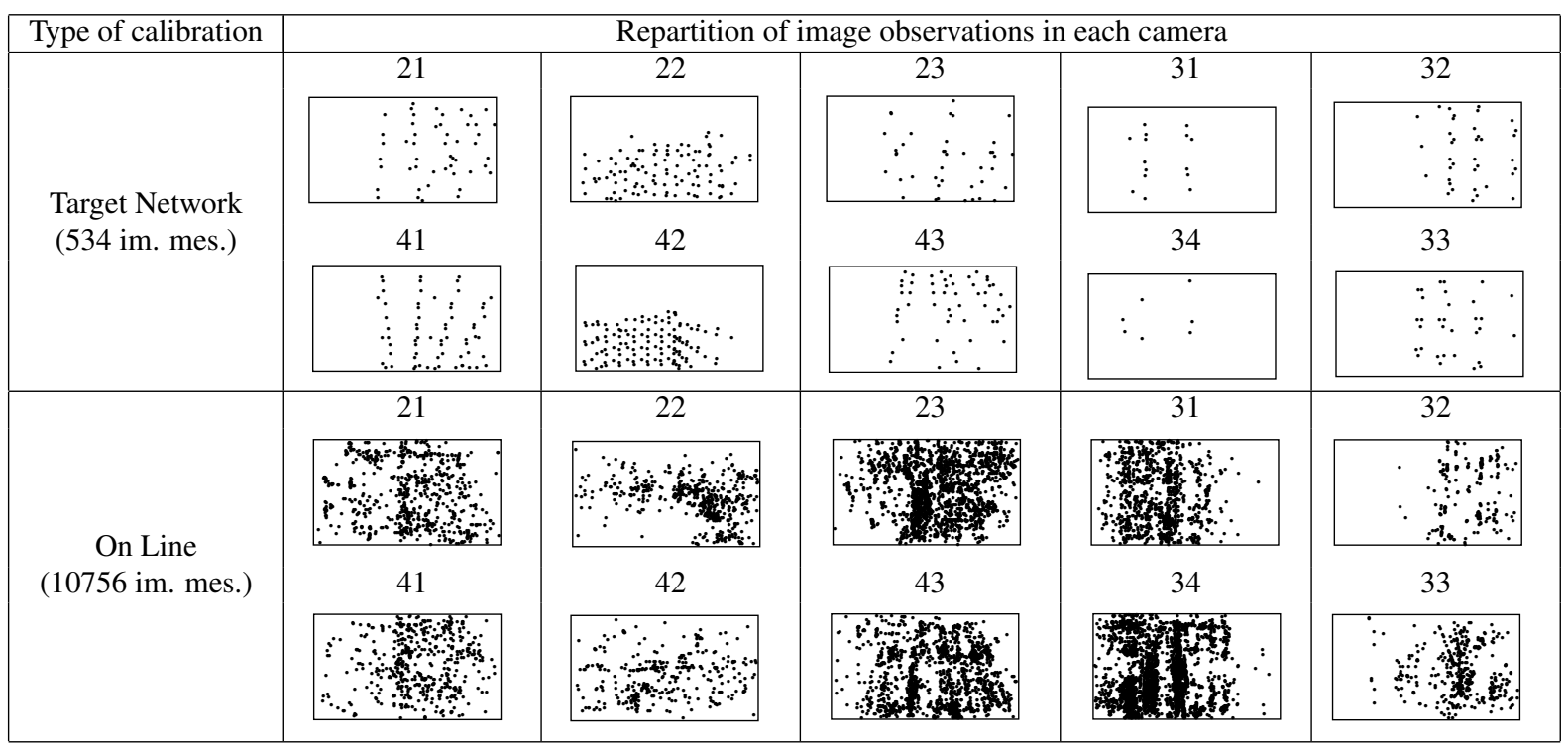

Table 4: Accumulation of image measurement on images plan. im. mes. (image measurments).

off-line and on-line calibration but on CPs, the on-line calibration is better which means that

Another interesting result concerns the distribution of measurements in image space. Table 4 shows the difference of density between two methods. The on-line method provides a much higher density and a better sampling of image space due to the accumulation of tie points coming from random lanscape contents.

\subsection{Pros and Cons}

Off-line calibration requires an topometric calibration facility, which is not so easy to build and to maintain. The calibration facility can also be far away from the survey sites and the offline calibration can thus be done in conditions very different from that of the surveys (e.g. difference in temperature which can have an impact on the mechanical deformation of the metal body on which all the sensors are attached).

On-line calibration use directly the surveys themselves. Thus on the one hand, the number of tie points is potentially much higher and on the other hand the spatial distribution of tie points sample the image space much more densely. One can see on table 4 the repartition of measurements in the different image planes for both calibrations.

The on-line calibration is very efficient and accurate in urban areas but can be unreliable if the environment is not friendly to extract tie points (e.g. forests, etc.).

\section{CONCLUSION AND PERSPECTIVE}

This paper has presented two complete methods to calibrate a multi-camera mobile mapping system. Although we have adressed the specific application of the calibration of a multi-camera panorama head with very low overlap between neighbouring images, the two methods presented can be used for any type of mobile platform and any type of multi-camera configuration. The self-calibration method is very interesting from an operationnal point of view, it is fully automatic, it does not need a topometric network, and it adapts itself to the conditions of each survey (mecanic effect oftemperature, etc.). Future work will consist to improve the quality of the on-line calibration by testing, comparing and finding optimal geometric configurations to realize the calibration in practice (L-shape, S-shape, loop, etc.) and analyze the evolution of the results with the numbers of images.

\section{REFERENCES}

Ellum, C. and El-Sheimy, N., 2002. Land-based mobile mapping systems. Photogrammetric Engineering Remote Sensing 68(1), pp. 13-17.

Hartley, R. and Zisserman, A., 2000. Multiple View Geometry in Computer Vision. Cambridge University Press.

Lowe, D. G., 2004. Distinctive image features from scaleinvariant keypoints. International Journal of Computer Vision 60(2), pp. 91-110.

McGlone, J., Mikhail, E., Bethel, J. and Mullen, R., 2004. Manual of photogrammetry (5th Edition). American Society for Photogrammetry and Remote Sensing.

Petrie, G., 2010. An introduction to the technology mobile mapping systems. GEOInformatics 13, pp. 32-43.

Scheller, S., Westfeld, P. and Ebersbach, D., 2007. Calibration of a mobile mapping camera system with photogrammetric method. International Archives of Photogrammetry, Remote Sensing and Spatial Information Sciences 36 (Part 5/C55), pp. on CD-rom.

Schmid, H., 1959. A general analytical solution to the problem of photogrammetry. Technical Report 1065, Ballistic Research Laboratories, US.

Triggs, B., McLauchlan, P., Hartley, R. and Fitzgibbon, A., 2000. Bundle adjustment - a modern synthesis. In: W. Triggs, A. Zisserman and R. Szeliski (eds), Vision Algorithms: Theory and Practice, LNCS, Springer Verlag, pp. 298-375.

Tsai, R. Y., 1987. A versatile camera calibration technique for high-accuracy $3 \mathrm{~d}$ machine vision metrology using off-the-shelf tv cameras and lenses. IEEE Journal of Robotics and Automation 3(4), pp. 323-344. 